\title{
Benefits and ethical issues in domino liver transplant
}

\author{
Nicolae Bacalbasa ${ }^{1,2}$, Irina Balescu ${ }^{3}$, Adina Croitoru ${ }^{4,5}$, Simona Dima $^{4}$, \\ Mihaela Vilcu ${ }^{1,2}$, Iulian Brezean ${ }^{1,2}$ \\ 1"Carol Davila” University of Medicine and Pharmacy, Bucharest, Romania \\ 2"Ion Cantacuzino" Clinical Hospital, Bucharest, Romania \\ 3"Ponderas" Academic Hospital, Bucharest, Romania \\ ${ }^{4}$ Fundeni Clinical Institute, Bucharest, Romania \\ ${ }^{5}$ Faculty of Medicine, "Titu Maiorescu" University, Bucharest, Romania
}

\begin{abstract}
Due to the permanent discordance between the number of available liver grafts and the number of cases on the waiting lists, attention was focused on developing different solutions in order to increase the number of grafts. Once the technique of living donor has been proposed, the number of grafts increased but the gap between the number of available grafts and the cases on the waiting list is still significant. Therefore attention was focused on finding out other solutions such as splitting the graft or performing a domino liver transplant. The procedure of domino transplant consists of harvesting a graft from a young patient with metabolic disorders in which a healthy graft will be used and implants it to a marginal receiver. The method seems to associate minimal risks for the marginal donor to develop the metabolic disorder especially if the donor suffers from familial amyloid polyneuropathy; however, ethical issues in such cases are obvious. The aim of this paper is to analyse both the benefits of domino liver transplant and the ethical issues of the procedure.
\end{abstract}

Keywords: domino liver transplant, metabolic disorders, survival, ethical issues

\section{INTRODUCTION}

Although the techniques of hepato-bilio-pancreatic surgery as well as those of liver transplantation significantly improved in the last decades, there is still an important gap between the number of donors and the number of cases necessitating a liver graft. Therefore, the per cent of cases who die while being on the waiting list remains extremely high; in order to find out a solution to decrease the number of patients who die before having the chance to be transplanted, various options have been proposed so far. The most commonly accepted ones are represented by harvesting a hemi liver from a living donor or splitting the graft for two donors; another option which has been proposed in the domino liver transplantation $(1,2)$.

\section{HISTORY OF THE CONCEPT OF DOMINO TRANSPLANT}

The concept of domino transplantation was imagined in the '90's by the cardiothoracic surgeons; therefore, they observed that a significant number of patients with end stage pulmonary disease who necessitate a heart-lung transplantation had themselves well-functioning hearts; in consequence, after performing the heart-lung transplantation, their hearts served as grafts for patients with end stage heart disease and with good pulmonary function (3). In such cases both the patient necessitating a heart-lung transplant as well as the one necessitating a heart transplant will receive certain well-functioning organs which will improve their quality of life as well as their lifespan. 
When it comes to the patients necessitating a liver transplant, most often they present locally advanced, unresectable malignancies, their lifespan in the absence of liver transplantation is minimal and, unfortunately an important part of them will die before having the chance to be transplanted. More recently, other indications for liver transplant have been proposed; therefore, in the early ' 90 's the first studies which explored the efficacy of liver transplant in children with inborn metabolism errors have been published. In certain cases the liver of these children is structurally normal and theoretically it could be transplanted itself to marginal recipients $(4,5)$. For example, at Karolinska University Hospital, domino liver recipients are chosen among patients presenting liver malignant tumors, among older than 40 years cases who are diagnosed with hepatitis-induced cirrhosis, among older than 60 years patients, irrespectively of the indication of transplant or among cases necessitating retransplantation after chronic graft failure (6). In all these cases it is considered that the risk of developing a metabolic disorder after a domino procedure is lower when compared to their life expectancy after liver transplant (7).

\section{RISKS OF DE NOVO DISEASE DEVELOPMENT IN RECIPIENTS AFTER DOMINO TRANSPLANTATION}

One of the most important factors which should be analysed when the decision of domino liver transplant is taken into consideration is related to the risk of the recipient of developing the disease of the donor. Therefore, most cases that are considered as domino donors are selected among hemodynamically stable children or adolescents with no signs of portal hypertension and a good general status. They are usually submitted to living donor liver transplantation while their liver is harvested and transplanted in a marginal recipient. In consequence, is mandatory to know which the chance is for the recipient of the domino graft to develop the disease. Most often the domino transplant donors will need few decades to become symptomatic, so the recipient may never develop the disease which might be induced by the aberrant metabolic functioning of a certain enzyme from the harvested domino liver graft.

An acceptable indication for domino liver donor is represented by patients diagnosed with familial amyloid polyneuropathy, an autosomal dominant neurodegenerative disease characterised by the presence of a mutation of transthyretin, causing in this way the precipitation of amyloid at the level of heart, kidney, central nervous system. It is estimated that the symptoms will develop after a median period of 35 years and that the process will evolve to an end stage disease 10-15 years after the onset of these symptoms. The rationale for which liver transplant is proposed in such cases is related to the fact that up to $90 \%$ of the circulating transthyretin is synthetized in the liver. The first successful transplant for this metabolic disorder was performed in 1991 and after this moment, multiple such procedures have been reported; in terms of domino transplant, it has been demonstrated that the symptoms of the disease will develop to the recipient of a domino liver graft within 7-9 years after transplantation $(3,8,9)$. Another metabolic disease which might be treated by a domino liver transplant is represented by familial hypercholesterolemia; however, in such cases, recipients of the domino grafts are at risk of developing drug refractory cardiovascular or atherosclerotic lesion, therefore long term follow-up is needed (3).

Analysing and comparing the outcomes of the domino liver transplant receivers to those of patients submitted to standard liver transplantation might be difficult due to the fact that most often the receivers of the domino liver grafts are usually older and have a more advanced stage of the disease (3).

\section{ETHICAL ASPECTS OF DOMINO TRANSPLANTATION}

Obviously, transplantation of a marginal liver graft which is harvested from a patient with serious metabolic issues is associated with serious ethical issues. Once the domino transplant is performed, the receiver will be at risk of developing at a certain moment of his evolution a similar metabolic disorder. Therefore, a long term follow-up is needed in the next years. According to the Familial Amyloidotic Polyneuropathy World Transplant Registry and the Domino Liver World Transplant Registry which are held at Karolisnka University, Sweden, among the 569 cases submitted to domino liver transplant worldwide only two cases developed the symptoms of familial amyloidotic polyneuropathy at eight years and seven years respectively after liver transplant; in the meantime the overall survival at one year, three years and five years follow-up were of $79.9 \%, 65.3 \%$ and $61.6 \%$ respectively (10). Another interesting study which was conducted on 
this theme in Sweden on 35 patients submitted to domino liver transplant between 1997 and 2007 demonstrated that the electroneurographic signs of polyneuropathy developed at 2-5 years after transplantation, none of these cases being symptomatically. Moreover, the one year survival rate was of $63 \%$ among patients submitted to liver transplant for hepatocellular carcinomas and $94 \%$ for nonhepatocellular carcinomas (6).

Another similar study was conducted in Portugal by Nunes et al. and included 41 cases submitted to domino liver transplant, with a median age of 53 years. The main pathology of the liver disease was represented by alcoholic cirrhosis followed by hepatitis $\mathrm{C}$ cirrhosis; performing a domino liver transplant leaded to an increase of the number of the grafts with up to $25 \%$ in a single center and with up to $16 \%$ at national level, while the risk of developing of clinical manifest familial amyloidotic neuropathy seemed to be minimal (11). Moreover, other authors underlined the fact that excellent results might be achieved after domino liver transplantation but special care should be taken in order to prevent the development of post-transplant thrombotic events (11).

However, when analysing the ethical issues of domino liver transplantation, it should not be omitted the fact that in the absence of the liver transplant the marginal receiver would experience a minimal survival rate, while after performing such a procedure the risk of developing a metabolic disorder could develop usually after a few years; moreover, this risk remains low, enabling the surgeon to consider the procedure to be rather an ethical one (7). Moreover, in certain centers it has been proposed that retransplantation from cadaveric donor should be considered if the receiver of a domino graft survives more than five years $(12,13)$. Once again this procedure proves to be an ethical one; in this way performing a domino transplant increases the life span of a patient who could otherwise die on the waiting list and offers him the chance to be submitted to retransplantation after a certain period of time, when a graft from a deceased donor is available (14).

Even if the risk to develop a metabolic disorder after domino liver transplantation seems to be re- duced, the recipient and its family should be carefully informed about these risks and should give their consent in order to proceed to this surgical gesture (11). Moreover, certain authors proposed creating a specific informed consent for such cases (15) through which the domino donor is assured that he will not be exposed to any supplementary risk while the domino receiver is informed about the risk of developing the metabolic disease at a certain moment. In the meantime, the Portuguese researchers also added in this type of consent the necessity of assuring the patient that he would have the opportunity to receive a "healthy" graft from a deceased donor at a certain moment (16).

Another important aspect which should be taken in consideration whenever a domino transplant is proposed is related to the manner in which the liver is harvested from the domino donor. In such cases the technique of liver harvesting should be carefully performed in order not to induce any additional risk (7).

\section{CONCLUSIONS}

Domino liver transplant seems to be an efficient method in order to prolong the life span for marginal recipients on the waiting list, the rate of developing metabolic disorders being relatively low. However, receivers of domino liver grafts should be submitted to long term follow up protocols in order to diagnose the possible metabolic disorders as soon as possible. Therefore, we can conclude that although domino liver transplant seem to offer a significant benefit in terms of survival, the ethical issues should be also taken in consideration and carefully analysed in each case before deciding to perform such a procedure.

\section{Acknowledgement}

This work was supported by the project entitled „Multidisciplinary Consortium for Supporting the Research Skills in Diagnosing, Treating and Identifying Predictive Factors of Malignant Gynecologic Disorders", project number PN-III-P1-1.2-PCCDI2017-0833.

\section{REFERENCES}

1. 2009 Annual Report of the U.S. Organ Procurement and Transplantation Network and the Scientific Registry for Transplant Recipients: 1999-2008 Transplant Data. Rockville, MD, U.S.: Department of

Health and Human Services, Health Resources and Services Administration, Healthcare Systems Bureau, Division of Transplantation; 2010. 
2. Klepetko W, Wollenek G, Laczkovics A et al. Domino transplantation of heart-lung and heart: an approach to overcome the scarcity of donor organs. J.Heart Lung Transplant. 1991; 10: 129-31.

3. Kitchens WH. Domino liver transplantation: indications, techniques, and outcomes. Transplant. Rev. (Orlando.) 2011; 25: 167-77.

4. Moini M, Mistry P, Schilsky ML. Liver transplantation for inherited metabolic disorders of the liver. Curr. Opin. Organ Transplant. 2010; 15: 269-76.

5. Hemming AW, Cattral MS, Greig PD et al. Domino liver transplantation for familial amyloid polyneuropathy: optimal use of a scarce resource. Transplant. Proc. 1999; 31: 515

6. Ericzon BG, Larsson M, Wilczek HE. Domino liver transplantation: risks and benefits. Transplant.Proc. 2008; 40: 1130-1.

7. Wilczek HE, Larsson $M$, Yamamoto $S$ et al. Domino liver transplantation. J.Hepatobiliary.Pancreat.Surg. 2008; 15: 139-48.

8. Holmgren G, Steen L, Ekstedt J et al. Biochemical effect of liver transplantation in two Swedish patients with familial amyloidotic polyneuropathy (FAP-met30). Clin.Genet. 1991; 40: 242-6.

9. Stangou AJ, Hawkins PN. Liver transplantation in transthyretin-related familial amyloid polyneuropathy. Curr.Opin.Neurol. 2004; 17: 615-20.
10. Ericzon BG. Domino transplantation using livers from patients with familial amyloidotic polyneuropathy: should we halt? Liver Transpl. 2007; 13: 185-7.

11. Nunes $F$, Valente $M$, Pereira $R$ et al. Domino liver transplant: influence on the number of donors and transplant coordination. Transplant.Proc. 2004; 36: 916-7.

12. Azoulay D, Samuel D, Castaing D et al. Domino liver transplants for metabolic disorders: experience with familial amyloidotic polyneuropathy. J.Am.Coll.Surg. 1999; 189: 584-93.

13. Hemming AW, Cattral MS, Chari RS et al. Domino liver transplantation for familial amyloid polyneuropathy. Liver Transpl.Surg. 1998; 4: 236-8.

14. Marin-Gomez LM, Gomez-Bravo MA, Barrera-Pulido L et al. Outcomes of domino liver transplantation: a single institution's experience. Transplant.Proc. 2010; 42: 644-6.

15. Schenck D, Mazariegos GV, Thistlethwaite JR, Jr. et al. Ethical Analysis and Policy Recommendations Regarding Domino Liver Transplantation. Transplantation 2018; 102: 803-8.

16. Furtado AJ. Domino liver transplantation using FAP grafts. HUC experience--hopes and realities. Amyloid. 2003; 10 Suppl 1: 84-7. 